\title{
SIMULATION OF TRAFFIC FLOW DURING EMERGENCY EVACUATIONS: A MICROCOMPUTER BASED MODELING SYSTEM
}

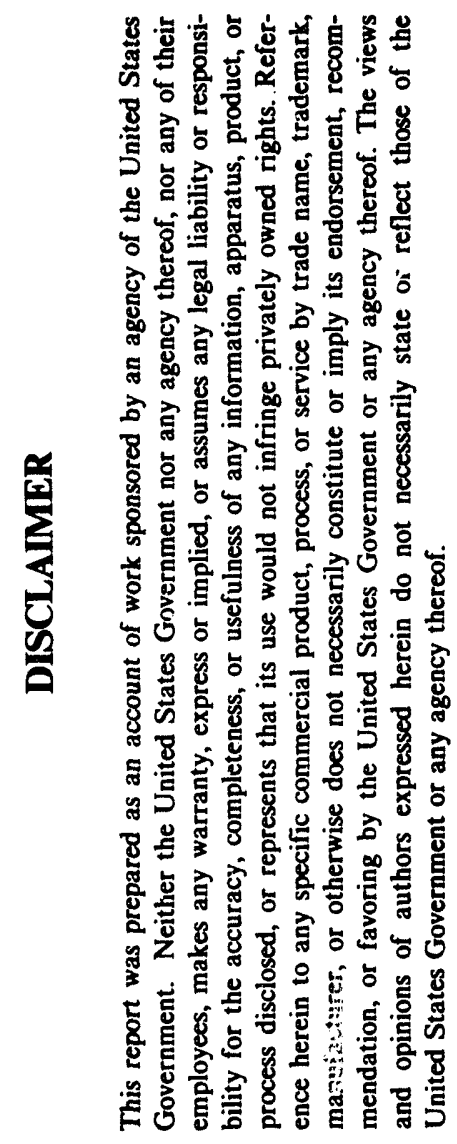

\author{
Ajay K. Rathi \\ Rajendra S. Solanki \\ IVHS Research Group \\ Center for Transportation Analysis \\ Oak Ridge National Laboratory
}

September 1993

Prepared by the

OAK RIDGE NATIONAL LABORATORY

Oak Ridge, Tennessee 37831

managed by

MARTIN MARIETTA ENERGY SYSTEMS, INC.

for the

U. S. DEPARTMENT OF ENERGY

under contract DE-AC05-84OR21400

"The submitted manuscript has been authored by a contractor of the U.S. Government under contract No. DE-AC05-84OR21400. Accordingly, the U.S. Government retains a nonexclusive, royalty-free license to publish or reproduce the published form of this contribution, or allow others to do so, for U.S. Government purposes."

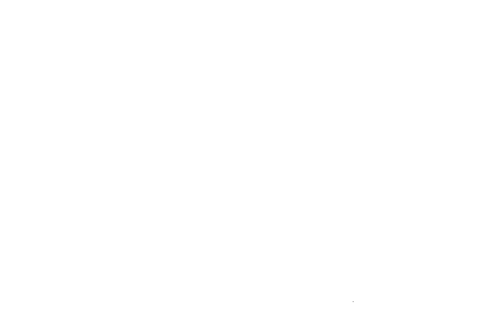




\title{
SIMULATION OF TRAFFIC FLOW DURING EMERGENCY EVACUATIONS: A MICROCOMPUTER BASED MODELING SYSTEM
}

\author{
Ajay K. Rathi \\ Rajendra S. Solanki \\ IVHS Research Group \\ Center for Transportation Analysis \\ Oak Ridge National Laboratory \\ P. O. Box 2008 \\ Oak Ridge, Tennessee 37831-6206
}

\begin{abstract}
Evacuation is one of the major and often preferred protective action options available for emergency management in times of threat to the general public. One of the key factors used in evaluating the effectiveness of evacuation as a protective action option is the estimate of time required for evacuation. The time required for evacuation is the time associated with clearing an area at risk to areas far enough away to be considered safe. Computer simulation models of traffic flow are used to estimate the time it takes to evacuate or "clear" an at-risk region by means of vehicular evacuation.

This paper provides a brief description of the Oak Ridge Evacuation Modeling System (OREMS), a prototype under development at the Oak Ridge National Laboratory. OREMS consists of a set of computer programs and models which can be used to simulate traffic flow during regional population evacuations and to develop evacuation plans for different events and scenarios (e.g. good vs. bad weather and nighttime vs. daytime evacuations) for user-defined spatial boundaries.
\end{abstract}

\section{INTRODUCTION}

The U.S. Army stockpiles unitary chemical weapons, both as bulk chemicals and as munitions, at eight major sites in the United States. The continued storage and disposal of the U.S. unitary chemical stockpile has the potential for accidental releases of toxic gases that could escape the installation boundaries and pose a threat to the civilian population in the vicinity. The U.S. Army, in conjunction with the Federal Emergency Management Agency (FEMA) and other federal agencies, is committed to implementing emergency preparedness program that will significantly reduce the adverse effects of accidental releases from the chemical stockpile (Carnes et al. 1989).
Evacuation is one of the major and often preferred protective action options available for emergency management in times of threat to the general public (Rogers et al. 1990). For the Chemical Stockpile Emergency Preparedness Program (CSEPP), evacuation by itself or in conjunction with other protective actions (e.g., respiratory protection) is being considered as a viable option to reduce the risk of adverse health effects from accidents involving the chemical agent stockpile (Carnes et al. 1989).

One of the key factors used in evaluating the effectiveness of evacuation as a protective action option is the estimate of time required for evacuation. The time required for evacuation is the time associated with clearing an area at risk to areas far enough away to be considered safe. Computer simulation models of traffic flow are used to estimate the time it takes to evacuate or "clear" an at-risk region by means of vehicular evacuation. In the United States, the IDYNEV computer model has been used to estimate evacuation times as part of emergency response plans for several nuclear power plants. The use of I-DYNEV has been endorsed by both the Nuclear Regulatory Commission (NRC) and Federal Emergency Management Agency (FEMA).

As part of a broad effort to provide technical assistance to FEMA and the U. S. ARMY for the CSEPP program, Oak Ridge National Laboratory (ORNL) has completed preliminary evacuation studies for all eight chemical stockpile storage sites. The objective of these preliminary Evacuation Time Estimate (ETE) studies was to generate estimates of the time it would take populations located both on base and within the vicinity of storage sites to evacuate should a significant chemical release occur for any reason. Evacuation studies were part of many technical studies performed under the CSEPP to support decision making and to form the basis of program guidance for the overall emergency preparedness program. A microcomputer version of the I-DYNEV model (named PCDYNEV) was used to estimate ETEs in these studies for a variety of 
scenarios. (See for example Rathi et al., 1991 and Metzger et al., 1991).

During the course of using I-DYNEV for evacuation studies for CSEPP, over 1000 executions of I-DYNEV were made by the ORNL project team. As a result of this rather extensive usage of I-DYNEV, ORNL team has identified several areas of enhancements that must be made if the model is to become a useful analysis tool for evacuation studies (Rathi et al. 1993). The enhancements include software enhancements as well as significant changes in model logic. These problems were observed in both the microcomputer PCDYNEV (KLD 1990) and the mainframe (IEM 1993) version of the model. These problems along with the need to develop a stand-alone, public-domain, microcomputerbased evacuation simulation model has motivated the development of the simulation modeling system described in the following sections.

\section{THE OAK RIDGE EVACUATION MODELING SYSTEM (OREMS)}

OREMS is a microcomputer-based system developed to simulate traffic flow during an emergency evacuation which may be undertaken in response to a conceivable man-made calamity such as nuclear reactor failures, airborne release of a toxic gas, and damfailure related flooding as well as a disaster brought on by a hurricane, an earthquake, or other natural causes.

OREMS consists of a set of computer programs and models which can be used to estimate evacuation time and to develop evacuation plans for different events or scenarios (e.g., good vs. bad weather conditions, day vs. nighttime evacuations) for user-defined spatial boundaries. OREMS allows the user to experiment with alternate routes, destinations, traffic control and management, and evacuee response rates. For a given situation, OREMS can help the planner identify the evacuation or clearance times, traffic operational characteristics (e.g., average speed), bottlenecks, and other information necessary to develop effective evacuation plans. All of the above information can be obtained for a given section of highway, an area within the network, a sector/ring, or at any other level of spatial aggregation. Detailed information on traffic operational characteristics can also be obtained at userspecified time intervals between the beginning and end ("clearing") of an evacuation.

\subsection{Capabilities of OREMS}

OREMS is designed to allow the user to perform comprehensive evacuation planning studies including estimates of evacuation times, development of traffic management and control strategies, identification of evacuation routes, identification of traffic control points, and other elements of an evacuation plan. There are six fundamental questions which OREMS can address:

- What is the time associated with evacuation (complete or partial) of the population at risk within the Emergency Planning Zone (EPZ)? The evacuation time estimates can also be obtained for specific sections of the highway network or subareas within the EPZ.

- How many people may be at risk based on the rate of evacuation? Once again, this information can be obtained for the entire EPZ or portions thereof. Specifically, OREMS can provide estimates of the number of vehicles still on the various segments of the highway network by time.

- What are the best route and path for the evacuation public from different areas of planning region?

- What is the best strategy for evacuating people as a protective option by itself or in combination with other protective action strategies?

- What are the best traffic management and control strategies?

- What could be the potential "hot spots" or "trouble spots" (in terms of traffic operations) within the EPZ?

It should be noted that many of the above answers are not "direct" output from the model. The information on evacuation time for the entire EPZ can readily be obtained from the output of the model. A post-simulation program (named SIMOD) can be used to estimate the clearance times for individual highway sections of the highways or communities within the EPZ in the context of evacuation of the entire region (not just the evacuation of the specific subarea of interest). The SIMOD program can also be used to obtain a number of other useful statistics associated with the evacuation, both as tables and as graphs. However, most of the above information must be intelligently "extracted" from the simulation output. In some instances, several iterations of the model may be required, for example when developing traffic control strategies.

Furthermore, at the present time OREMS is a standalone evacuation planning tool. It is not yet integrated with other related models such as PADRE or D2PC (CSEPP Progress Report, 1993). It is also not yet just a part of other larger emergency management information systems. Thus, the system is not capable of performing many other tasks required in developing evacuation plans. Many data items, which might be considered as output of the OREMS, are actually the required input to the model. The user must run other 
models or obtain data from other sources to develop inputs for the OREMS system. For example,

- OREMS does not estimate the timing of people's response to the perceived emergency by location.

- OREMS does not estimate the traffic control settings and parameters.

- OREMS does not estimate traffic demand (number of evacuees and evacuating vehicles by location).

- OREMS does not determine the EPZ

\subsection{OREMS's Components}

OREMS consists of three related computer programs, or execution files, accessed from a common graphical user-interface (see Figure 1). These programs of the OREMS perform three operations: input data file and management, analysis, and output displays. These component programs are:

- ESIM, a network traffic flow simulation model,

- IEVAC, an interactive graphical input data manager for ESIM, and

- SIMOD, an interactive graphical output display software for ESIM.

Given below is a brief description of major components of the OREMS.

\subsubsection{ESIM Traffic Analysis System}

ESIM (for Evacuation SIMulations) is the analytical core of OREMS. ESIM is a rather complex, FORTRAN-based program that simulates the traffic conditions over a transportation network as an evacuation progresses. The ESIM model combines a trip distribution and traffic assignment model with a detailed traffic flow simulation model. Through traffic distribution and assignment, ESIM determines the destinations selected by evacuees and the routes taken to reach the selected destinations. It also performs a detailed simulation of traffic operations on the evacuation network given these projected flows and routes under prevailing roadway and traffic conditions. This simulation allows the analyst to estimate service rates in the evacuation network by location and by time; identify performance characteristics of traffic; identify bottlenecks; and estimate evacuation times across various categories (link, sector, or region specific estimates by time).

The algorithms used in ESIM traffic assignment and simulation models are based on techniques that have resulted from years of research sponsored by the Federal Highway Administration (Lieberman et al. 1980). ESIM provides a reasonable estimate of evacuation time under prevailing conditions as described in the input data by the user.

\section{ESIM's Simulation Model}

ESIM's simulation model is an adaptation of the NETFLO II model of the TRAF family. The simulation model of NEIFLO II itself is a refinement and extension of the traffic flow model of the popular signal optimization program, TRANSYT (1980). TRANSYT incorporates a macroscopic simulation model based on platoon dispersion theories of traffic flow. The term macroscopic means that the model simulates the flow of traffic in some aggregate fashion rather than the movement of individual vehicles in a microscopic fashion. The analytical developments and details of the NETFLO II simulation model are described in an FHWA report (Lieberman et al. 1980).

In brief, the traffic stream in the NETFLO II model is represented in the form of movement-specific statistical histograms. The length of time during which simulation is performed is split up into a series of Time Periods (TP) with each TP being further subdivided into a series of Time Intervals (TI).

Traffic congestion and spillbacks are treated explicitly in the simulation model. Vehicles can move on to the receiving link only if there is space available to accommodate the vehicles that want to enter that link. In this way queues develop. The effects of traffic control measures (i.e., signals, STOP and YIELD Signs) are also simulated at every intersection. Vehicle routes are determined by the turn percentages obtained from traffic assignment and distribution models.

\section{ESIM's Traffic Distribution or Destination Selection Model}

The destination selection models are commonly referred to as the trip distribution models by transportation planners/analysts. Simply stated, trip distribution is the process by which origin and destination ends of a trip are defined. In the case of evacuation modeling, the origins of trips are determined based on the location of populations (in various categories) at the time of the emergency. The destinations selected by evacuees are modelled in one of the three ways:

1) evacuees will exit via pre-specified destinations based on an established evacuation plan,

2) evacuees will exit the 'at-risk area' by heading for the nearest destination in terms of distance or time, and 3) evacuees will exit via the closest destination in terms of time or distance on the basis of traffic conditions at their time of departure.

ESIM allows the user to specify the destinations associated with each traffic origin. As an option, the 


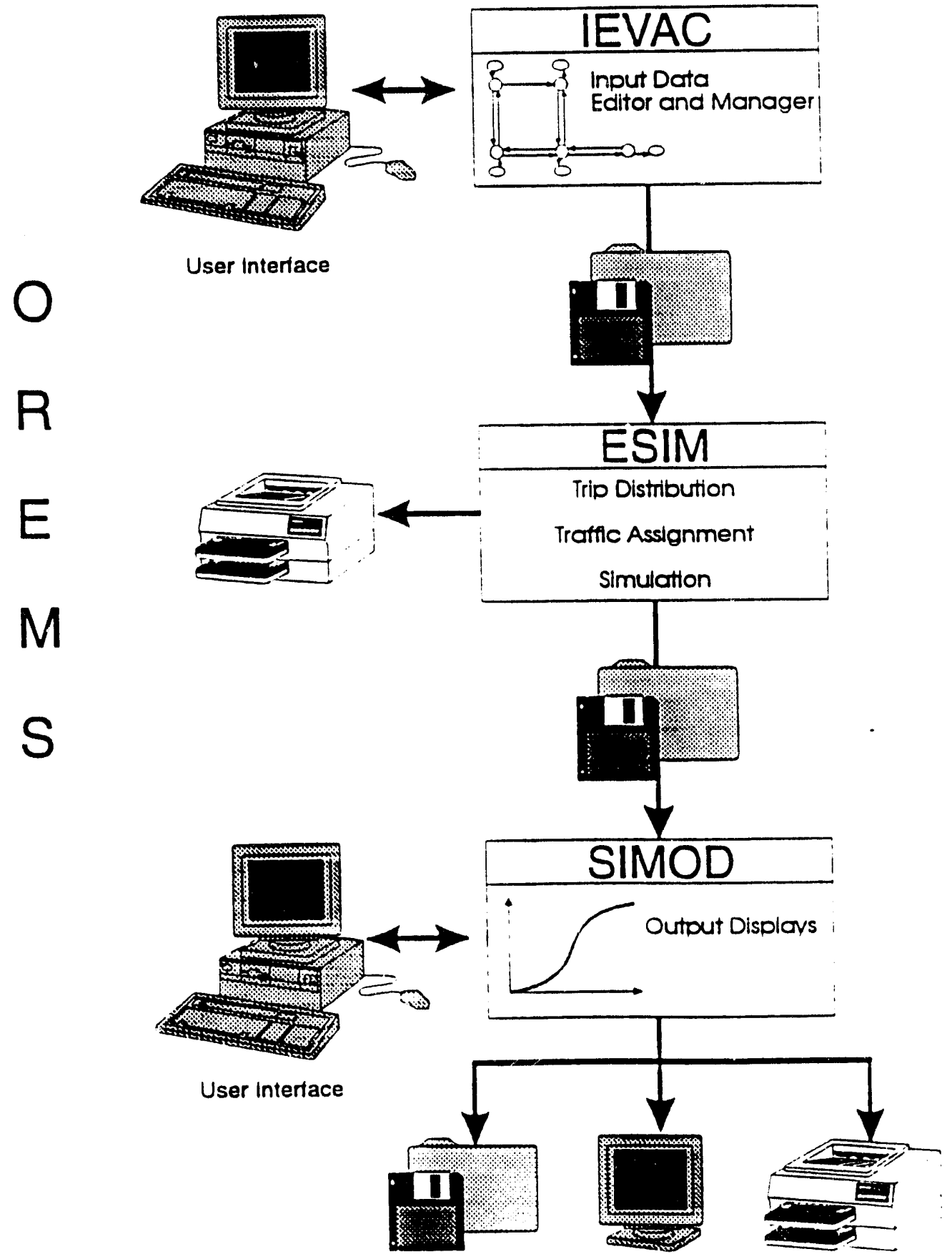

Figure 1 The Structure of OREMS 
model performs a trip distribution to determine the destinations for an origin. The destination selection (trip distribution) is based on a hybrid of the three modeling options described above.

The trip distribution algorithm of the ESIM model works as follows. Given the traffic volumes leaving each origin or source, and a list of its potential destinations, the algorithm distributes the flow accordingly (i.e., finds out which flows will go where and via which path). Note that the model in its current form deals with aggregate flows rather than individual vehicles.

To distribute traffic (i.e., assign destination), ESIM augments the geometric network by adding a set of pseudo-links and supernodes. The use of supernodes to solve the traffic distribution problem is well known and a common procedure (Sheffi 1985). By assigning different impedances to the pseudo-links connecting the different destinations to the supernodes, the algorithm will distribute traffic according to the enroute travel time and the assigned impedance. In other words, destinations with low assigned pseudo-link impedance will attract more traffic. In many situations, if there is no preference for specific destinations, an equal impedance will be assigned to all the pseudo-links. In this case, enroute travel time is the sole factor in distributing traffic. That is, more traffic will arrive at the closest destination, which may not be an unreasonable assumption.

\section{ESIM's Traffic Assignment Model}

Route selection models are used to approximate the path selected by evacuees (i.e., evacuation routes). The process of assigning paths to the traffic flow is referred to as traffic assignment in transportation planning. Traffic assignment models attempt to simulate the traffic flow on a transportation network over time, using the travel demand, travel behavior of drivers, and traffic conditions. The basic methodology in traditional traffic assignment is to determine a logical path between an origin and a destination under given traffic conditions, based on system or user optimal travel behavior. The results of the traffic assignment process are traffic flows on each link of the transportation network over time as well as average travel times associated with these flows.

ESIM's traffic assignment model is an equilibrium assignment model which attempts to find a user optimal solution. The term equilibrium is characterized by Wardrop's famous first principle (1952) which states that "the journey times on all routes actually used are equal, and less than those which would be experienced by a single vehicle on any unused route." For this user-optimal assignment principle, an equivalent minimization problem can be formulated. The solution to this problem thus produces the traftic pattern which satisfies Wardrop's first principle. The traffic assignment model of ESIM and TRAF is an elaboration of the equilibrium traffic assignment model, TRAFIC, developed by Nguyen (1975).

TRAFIC still is one of the best traffic assignment models. One of the key inputs to traffic assignment models is the estimate of the capacity of highway segments. Most if not all other traffic assignment models employ constant, estimated values of link capacities. It is well known that link capacity is a function of many factors including the unknown turn volumes on all approaches serviced by an intersection. A very comprehensive capacity estimation model has been included in the TRAF and thus ESIM models. This model produces, through iterations, accurate estimates of scrvice rates (capacities) for each link of the highway network by taking into account the assigned volumes and type of traffic control at each intersection. The solution procedure used in the capacity model is rapid, accurate, and unconditionally convergent.

\subsubsection{IEVAC Input Data Manager}

Simulation models by their very nature are data intensive and OREMS is no exception: it is perhaps an extreme example. The input data requirements for the ESIM model are rather extensive. To use the ESIM model for estimating evacuation time, the physical traffic environment must be specified by the user as input data. This input data describes:

- Topology ("connectivity") of the roadway system;

- Roadway characteristics including geometrics and channelization of traffic;

- Traffic volumes and composition;

- Traffic control devices and their operational characteristics;

- Origin-to-destination trip-table; and

- Vehicle/Driver performance characteristics.

To provide an efficient framework for defining these inputs, the roadway system is represented as a network of links and nodes. The links represent unidirectional roadway segments and the nodes represent intersections or points along the roadway where highway geometry changes. The network input data for ESIM consists of a series of logically sequenced records defining the required data as either link or node specific data items. In addition, several other input parameters must be specified for run control (i.e., simulation time) and run information. The user can change the default values embedded in the model by providing optional input. 
Table 1 summarizes the major input requirements of the ESIM model.

A typical input data file for ESIM consists of several hundred lines of data. The data structure is such that the user must adhere to very strict formats while utilizing countless numeric codes. Preparing the data files needed to run ESIM for even small networks can be a very tedious and time-consuming activity. IEVAC (for Interactive data manager for EVACuation modeling) is a graphics program which was developed to greatly simplify the task of creating the data base (or datafiles) necessary to use the ESIM simulation model. The program allows the user to create an input data filefrom scratch or modify an existing data file. IEVAC has been designed to simplify the task of data entry so that the user does not have to understand and remember the specific traffic model terminology and data structure used in ESIM data files. In addition, IEVAC performs comprehensive and intelligent error checking to insure the accuracy and consistency of the data. IEVAC is based on popular WYSIWYG (what you see is what you get) philosophy and has been designed to give the look and feel of a Windows ${ }^{\mathrm{TM}}$ application. Due to its total graphical mode of data entry and manipulation, IEVAC provides better functionality and performance relative to text-based data editors. IEVAC has over 100 functions which allow the user to oreate/edit data files efficiently. These functions handle file management, user interaction, display, data entry/manipulation, network sizing, printing, and various other options available with the program. The user can create or edit a data file without lifting a finger from the mouse.

Table 1. Input Requirements for the ESIM Model

\section{GEOMETRICS}

Link lengths.

Number of lanes (up to 6).

Number of lanes in turn pockets.

Grade.

Network connectivity defined in terms of target nodes for each receiving link.

\section{TRAFFIC VOLUMES}

On all entry links and sink/source nodes stratified by vehicle type: auto, car pool, bus, truck.

Link-specific turn movements or O-D matrix (Trip Table).

\section{TRAFFIC CONTROL SPECIFICATIONS}

Traffic signals timings.

Stop and Yield signs.

Right-turn-on-red (RTOR).

Route diversion specifications.

Turn restrictions.

Lane control (i.e., lane closure).

\section{DRIVER'S ANDOPERATIONSCHARACTERISTICS}

Driver's (vehicle-specific) response characteristics: aggressiveness, discharge headway.

Link-specific mean speed for free-flowing (unimpeded) traffic.

\subsubsection{SIMOD Output Display Program}

SIMOD (for SIMulation Output Displays) is an interactive graphics program which displays the input data to, and the results produced by, the ESIM evacuation simulation model. The data produced by the ESIM simulation model allow the user to analyze traffic conditions during a regional population evacuation. The model produces data on a variety of measures of effectiveness at user-specified time intervals. The statistics are provided for individual links as well as for the entire network in a summary format (see Table 2).

Table 2. Measures of Effectiveness output by ESIM
Measure

Travel

Moving time

Delay time

Total travel time

Mean travel time per vehicle

Mean delay per vehicle

Mean delay per vehicle-mile

Mean speed

Mean occupancy

Mean saturation

Vehicle stops

\section{$\underline{\text { Units }}$}

Vehicle-Miles and Vehicle Trips

Vehicle-Minutes

Vehicle-Minutes

Vehicle-Minutes

Seconds

Seconds

Seconds/Mile

Miles/Hour

Vehicles

Percent

Percent
These data are provided for each network link and are also aggregated over the entire network. Of course, the simulation ends at the user specified time or when the network is void of vehicles. 
Because of the detail of the output data, one could expend considerable time and energy trying to properly interpret the model output to derive statistics of interest. To understand and analyze the results of the simulation of population evacuations and resulting traffic conditions over the transportation network, the user must examine the output files created by ESIM. Typically these output files are several hundred pages of computer printout. The size of the output files is dependent upon the size (area) and characteristics (rural vs. urban) of the transportation network and the output options requested by the user. The output is in the form of statistics which are hard to visualize to even the most competent and experienced users. Furthermore, the output shows a few obvious statistics on the traffic conditions and the progress of evacuation for the entire area; it requires corsiderable additional computation to obtain sector-or area-specific statistics or information on bottlenecks in the transportation network.

The post-processor component of OREMS has been designed not only as a utility for analyzing the simulation output, but also to assist the user with evecuation planning. SIMOD includes a graphical representation of the simulated traffic conditions for a scenario and displays the "hot spots" in the network, area-specific statistics, and other i:seful information with a few clicks of the mouse. In summary, SIMOD provides the user a simple and effective means of reviewing ESIM input data and analyzing ESIM results.

\section{APPLICATION OF OREMS IN EVACUATION STUDIES}

The first step in evacuation planning and analysis, of course, is the delineation of the emergency planning zones. A three-zone concept for the application of evacuation and other protective action strategies is commoniy utilized in the planning process. These zones are: Immediate Response Zone (IRZ) which is the area closest to the point source of the potential disaster where prompt and effective response is most critical; Protective Action Zone (PAZ) is an area which is slightly farther away but still under a potential threat depending upon the type of accident and weather condition; and Precautionary Zone $(\mathrm{PZ})$ is the outermost boundary beyond which no adverse effect can be expected as a result of the disaster and therefore does not require any significant pre-event planning. For a given area, the specific zonal boundaries are determined on the basis of political, human, and topological factors, with spatial and temporal distribution of the hazard as the most important consideration.

Having determined the IRZ, PAZ, and $\mathrm{PZ}$ boundaries, an accurate and reasonably detailed representation of the highway network within these zones is needed to estimate evacuation times and to develop evacuation plans using OREMS. In addition, the following information is required:

a) estimate of traffic demand (number of evacuees and evacuating vehicles by location).

b) trip generation time (timing of people's response to the perceived emergency by location).

c) destination and route selection by evacuees.

d) capacity of the highway network. Capacity is defined as the maximum number of vehicles that could pass a given section of highway in one direction during a given time period under prevailing roadway and traffic conditions. Simply stated, capacity represents the maximum traffic flow rate in various sections of the highway network. At the most fundamental level, evacuation time studies involve a comparison of the evacuation traffic demand with the capacity (or service rate) of the highway network within an "at risk region".

Using the information in items (a) and (b) as input to a reasonably detailed description of the highway infrastructure, OREMS can be used to generate the information required in items (c) and (d). The traffic assignment/distribution model of ESIM determines the destination selected by evacuees and the route taken to reach the selected destinations. The simulation model component of ESIM performs a detailed simulation of traffic operations on the evacuation network given these projected flows and routes under prevailing roadway and traffic conditions. This simulation allows the analyst to estimate service rates in the evacuation network by location and by time, identify performance characteristics of traffic, bottlenecks, and estimate evacuation times across various categories (link-, areaor network-specific estimates by time). The user can specify the origin-destination flows rather than using the traffic distribution model. In most cases, traffic assignment followed by simulation must be performed to analyze the traffic operations during evacuations. Typically, the OREMS model has to be used in an iterative manner to obtain the best estimate of evacuation time. Iterations are required not only when experimenting with alternate control strategies but also to accommodate some limitations of the traffic model. 


\section{HARDWAREAND SOFTWAREREQUIREMENTS}

The first release of OREMS works only for IBMcompatible microcomputers under the DOS 5.0 or later versions of the operating system. In addition to the programs and other files supplied with this document, OREMS requires the following hardware and software: - An IBM-compatible microcomputer with an 80386 or higher preprocessor. The memory requirement is at least $8 \mathrm{MB}$ of RAM. The programs and data for OREMS will need $16 \mathrm{MB}$ of hard disk space to run efficiently. As you generate more scenarios, more disk space will be needed if the input and output data files are saved in the hard disk.

- DOS Version 5.0. OREMS can be run under IBM OS/2.0 or under a Windows operating system as a DOS application.

- A 13" or larger monitor with VGA or higher resolution is required, as is a mouse.

- A printer is needed if you want to make a hard copy of the displays included in the user interface, including the simulation results.

\section{AVAILABILITY AND WORK IN PROGRESS}

OREMS is being developed by the Center for Transportation Analysis in the Energy Division of the Oak Ridge National Laboratory under funding from the Federal Emergency Management Agency (FEMA) and the US Department of Army under the Chemical Stockpile Emergency Management Program (CSEPP). Version 1.0 of the OREMS is ready and is expected to be made available to interested users by the end of this year.

In its current form, OREMS is more user-friendly than I-DYNEV and also a better traffic model. However, much work still has to be done to make it a realistic traffic simulation model, to make the software more user-friendly and versatile, to integrate the model with other analysis tools such as a plume dispersion model, and to improve its computational efficiency.

Work is now in progress to:

1) test and debug OREMS;

2) improve user interfaces;

3) experiment with microscopic simulation models;

4) integrate traffic simulation, assignment and distribution models;

5) integrate the model with other planning models and PADRE;

6) develop versions of OREMS on other operating systems (i.e., Windows).

\section{REFERENCES}

Carnes S. A., et al. 1989(a). Site-Specific Emergency Response Concept Plans For The Chemical Stockpile Disposal Program: A Comparative Study. ORNL/TM-11357. Oak Ridge National Laboratory, Oak Ridge, TN 37831.

CSEPP, ORNL 1993. Financial/Technical Report for July. Oak Ridge National Laboratory, Oak Ridge, TN 37831.

IEM, Inc. 1993. Final Onpost-Offpost CSEPP Automation System (FEMIS): Requirements Analysis INTERIM DRAFT. Prepared for the U.S. Army and Nuclear and Chemical Agency.

KLD Associates, Inc. 1984. Formulations of the DYNEV and I-DYNEV Traffic Simulation Models

Used in ESF. Prepared for the Federal Emergency Management Agency. Washington, DC.

Lieberman, E. et al. 1980. Macroscopic Simulation for Urban Traffic Management - The TRAFLO Model, Volume l:Executive Summary: Development of TRAFLO Programs. Federal Highway Administration Report No. FHWA-RD-80-113.

Metzger, David N. et al. 1991. Evacuation Time Estimates for A nniston-A rmy Depot and Vicinity, Draft Report, ORNL. Oak Ridge National Laboratory, Oak Ridge, TN 37831.

Nguyen, S. and L. James 1975. TRAFIC: An Equilibrium Traffic A ssignment Program. University of Montreal.

KLD Associates, Inc. 1990. PC-DYNEV User's Manual. Huntington Station, NY.

Rathi, A. K. et al. 1993. User Manual for Oak Ridge Evacuation Modeling System (OREMS). Draft Report, ORNL. Oak Ridge National Laboratory, Oak Ridge, TN 37831.

Rathi, A. K. et al. 1991. Evacuation Time Estimates for Lexington-Blue Grass A rmy Depot and Vicinity, Draft Report, ORNL. Oak Ridge National Laboratory, Oak Ridge, TN 37831.

Rogers, G. O. et al. 1990. Evaluating Protective Actions for Chemical Agent Emergencies. Oak Ridge National Laboratory, ORNL-6615, Oak Ridge, TN 37831.

Southworth, Frank et al. 1990. Evacuation Time Estimates for Aberdeen Proving Ground and Vicinity. Draft Report, ORNL. Oak Ridge National Laboratory, Oak Ridge, TN 37831.

Sheffi, Y. $1985 . \quad$ Urban Transportation Networks.Prentice Hall, Inc., Englewood Cliffs, N.J.

Wardrop, J. G. 1952. Some Theoretical A spects of Road Traffic Research. Proceedings of the Institute of Engineering, Part II.1. 


\section{AUTHOR BIOGRAPHIES}

AJAY K. RATHI is a Group Leader for Intelligent Vehicle Highway Systems (IVHS) research at the Center for Transportation Analysis at Oak Ridge National Laboratory. He received his Ph.D. (1983) and M.S. (1980) in Civil Engineering at Ohio State University and he received his B.E. (1978) in Civil Engineering at Ravishankar University.

RAJENDRA S. SOLANKI is a Research Staff member for Intelligent Vehicle Highway Systems (IVHS) for the Center for Transportation Analysis at Oak Ridge National Laboratory. He received his Ph.D. (1986) in Systems Analysis at Johns Hopkins University. He received his M.S. (1980) in Environmental Engineering at Indian Institute of Technology, and received his B.S. (1978) in Civil Engineering at the University of Roorkee. 


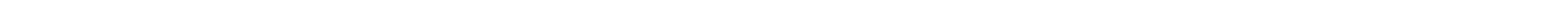




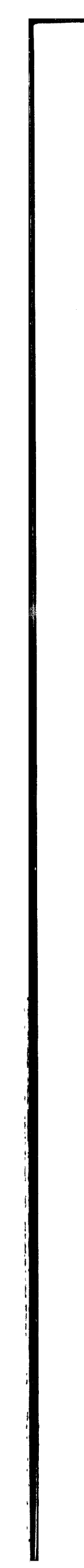

